\title{
Short communication: Effect of oxygen on symbiosis between Lactobacillus bulgaricus and Streptococcus thermophilus
}

\author{
H. Horiuchi ${ }^{* 1}$ and Y. Sasaki† \\ ${ }^{*}$ Research and Development Laboratories, and \\ †Food Science Institute, Meiji Co. Ltd., 540 Naruda, Odawara, Kanagawa 250-0862, Japan
}

\begin{abstract}
Lactobacillus delbrueckii ssp. bulgaricus (L. bulgaricus) and Streptococcus thermophilus are traditionally used for the manufacture of yogurt. It is said that a symbiotic relationship exists between Strep. thermophilus and $L$. bulgaricus and this decreases fermentation time. It is well known that L. bulgaricus is stimulated by the formate produced by Strep. thermophilus, and Strep. thermophilus is stimulated by free amino acids and peptides liberated from milk proteins by $L$. bulgaricus in symbiotic fermentation. We found that acid production by starter culture LB81 composed of L. bulgaricus 2038 and Strep. thermophilus 1131 was greatly accelerated by decreasing dissolved oxygen (DO) to almost $0 \mathrm{mg} / \mathrm{kg}$ in the yogurt mix (reduced dissolved oxygen fermentation) and that DO interferes with the symbiotic relationship between $L$. bulgaricus 2038 and Strep. thermophilus 1131. We attributed the acceleration of acid production of LB81 by reduced dissolved oxygen fermentation mainly to the acceleration of formate production and the suppression of acid production of LB81 by DO mainly to the suppression of formate production.
\end{abstract}

Key words: symbiotic relationship, Lactobacillus bulgaricus, Streptococcus thermophilus, dissolved oxygen

\section{Short Communication}

Fermentation is one of the oldest methods for extending the shelf life of milk (Tamime and Robinson, 1999). The definition of yogurt by the Food and Agriculture Organization of the United Nations/World Health Organization (FAO/WHO) standards is "Yogurt is the coagulated milk product obtained by lactic acid fermentation through the action of Lactobacillus delbrueckii ssp. bulgaricus (L. bulgaricus) and Streptococcus thermophilus from milk and milk products. The microorganisms in the final product must be viable

Received November 14, 2011

Accepted February 2, 2012.

${ }^{1}$ Corresponding author: hiroshi.horiuchi@meiji.com and abundant" (FAO/WHO, 1984). Such a product is beneficial to human health (Tamime and Robinson, 1999). Michaylova et al. (2007) suggested that Strep. thermophilus and L. bulgaricus strains widely used for commercial yogurt production could have originated from plants in Bulgaria.

Streptococcus thermophilus and L. bulgaricus are traditionally used for the manufacture of yogurt (HerveJimenez et al., 2009). In a mixed culture, acid production is much larger than the sum of a single culture (Driessen et al., 1982). The 2 bacterial strains in yogurt (L. bulgaricus and Strep. thermophilus) stimulate each other during their associative growth (Veringa et al., 1968). Perez et al. (1991) suggested that the symbiotic relationship between Strep. thermophilus and L. bulgaricus is very important for yogurt and cheese production because it decreases fermentation time. The stimulation of lactobacilli is caused mainly by the production of formate by streptococci. Veringa et al. (1968) suggested that the substance that stimulates L. bulgaricus and that is produced by Strep. thermophilus in yogurt milk is formic acid. Suzuki et al. (1986) suggested that formic acid was the only effective substance for the active growth of $L$. bulgaricus among purine ring precursors. Driessen et al. (1982) suggested that, in yogurt, the growth of Strep. thermophilus is stimulated by free AA and peptides liberated from the milk proteins by L. bulgaricus. Galesloot et al. (1968) suggested that the more proteolytic L. bulgaricus liberates in milk certain AA, which stimulates Strep. thermophilus.

The yogurt starter cultures of $L$. bulgaricus and Strep. thermophilus, are facultatively anaerobic, so the fermentation of yogurt with these bacteria progresses well in the presence of oxygen. The majority of strains of lactic acid bacteria, including the 4 genera Streptococcus, Leuconostoc, Pediococcus, and Lactobacillus, are aerotolerant to some degree (Condon, 1987). Sakamoto and Komagata (1996) reported that most of the 22 strains of lactic acid bacteria, including the genera Lactobacillus, Pediococcus, Leuconostoc, Streptococcus, and Enterococcus, grow well under aerobic conditions. The effect of the initial level of dissolved oxygen (DO) on acid production in buffalo milk by different single lactic 
cultures of Streptococcus lactis, Streptococcus diacetylactis, Streptococcus cremoris, L. bulgaricus, and Strep. thermophilus was tested by Shekar and Bhat (1983). They reported that the rates of acid production in buffalo milk by these lactic cultures increased with the decrease of initial oxygen content from 5.50 to $2.90 \mathrm{mg} /$ $\mathrm{kg}$, and that incorporating oxygen into milk to raise the initial oxygen content from 5.50 to $9.00 \mathrm{mg} / \mathrm{kg}$ strongly inhibited acid production. Kawasaki et al. (2006) investigated the effects of oxygen on the Bifidobacterium species using liquid shaking cultures under various oxygen concentrations. They reported that although most of the Bifidobacterium species showed oxygen sensitivity, 2 species (B. boum and B. thermophilum) showed growth stimulation in the presence of oxygen. Driessen et al. (1982) suggested that L. bulgaricus Ib needed carbon dioxide produced by Strep. thermophilus for optimal growth in milk. Although there have been many studies on the influence of oxygen on lactic acid bacteria, only a few studies have reported on the influence of oxygen on the symbiosis between L. bulgaricus and Strep. thermophilus.

The yogurt starters L. bulgaricus and Strep. thermophilus are well-known facultative anaerobic bacteria that can grow in oxygenated environments. Starter culture LB81, composed of L. bulgaricus 2038 and Strep. thermophilus 1131, reduced the DO in the yogurt mix before acid formation. The starter began to produce acid actively after the DO concentration in the yogurt mix had been reduced to almost $0 \mathrm{mg} / \mathrm{kg}$. Fermentation with LB81 was suppressed by the presence of more than $1 \mathrm{mg}$ of $\mathrm{DO} / \mathrm{kg}$ in the yogurt mix. However, acid production by L. bulgaricus 2038 or Strep. thermophilus 1131 alone was neither suppressed nor advanced by the level of the DO concentration in the yogurt mix. It was suggested that DO interferes with the symbiotic relationship between L. bulgaricus and Strep. thermophilus (Horiuchi et al., 2009). From our previous work, it was investigated that acid production by LB81 was suppressed greatly with $1 \mathrm{mg} / \mathrm{kg}$ or more of DO in the yogurt mix and was promoted with $0 \mathrm{mg} / \mathrm{kg}$; however, acid production by L. bulgaricus 2038 or Strep. thermophilus 1131 alone was neither suppressed nor advanced by the level of DO concentration in the yogurt mix (Horiuchi et al., 2009). From those results, we hypothesized that DO interferes with the symbiotic relationship between L. bulgaricus and Strep. thermophilus. In this work, we investigated the influence of oxygen on the symbiosis between L. bulgaricus and Strep. thermophilus, aiming at growth promoting factors for formic acid. In the discussion, we shall present a mechanism inference for fermentation abridgement by reduced dissolved oxygen fermentation (ROF) from our results, aimed at DO and formic acid.
Yogurt starter cultures of L. bulgaricus 2038 and Strep. thermophilus 1131 were used in this study. These strains were obtained from the culture collection of the Food Research and Development Center, Meiji Dairies Corp. (Tokyo, Japan). The combination of L. bulgaricus 2038 and Strep. thermophilus 1131, named LB81, and these single cultures were used in this study. Starter culture LB81 has been used in the commercial production of Meiji Bulgaria Yogurt LB81 in Japan since 1993.

Each strain of L. bulgaricus 2038 or Strep. thermophilus 1131 , stored at $-80^{\circ} \mathrm{C}$, was subcultured once at $37^{\circ} \mathrm{C}$ for $16 \mathrm{~h}$ in a skim milk and yeast extract medium composed of $10 \%$ (wt/wt) skim milk supplemented with $0.1 \%$ (wt/wt) yeast extract (preculture, single starter) after autoclaving (at $121^{\circ} \mathrm{C}$ for $7 \mathrm{~min}$ ), and was cooled to $5^{\circ} \mathrm{C}$. Both precultures of L. bulgaricus 2038 and Strep. thermophilus 1131 were inoculated (1\%; wt/ wt) into a fresh, sterilized skim milk and yeast extract medium (at $95^{\circ} \mathrm{C}$ for $10 \mathrm{~min}$ ), incubated at $37^{\circ} \mathrm{C}$ to reach an acidity of $0.7 \%$, and cooled immediately to $5^{\circ} \mathrm{C}$ (yogurt bulk starter culture LB81). The acidity was measured by titrating a 9 -g sample against $0.1 \mathrm{~N}$ sodium hydroxide, using phenolphthalein as the indicator. The yogurt starter culture was then stored at $5^{\circ} \mathrm{C}$. The yogurt starter culture LB81, which was maintained at $5^{\circ} \mathrm{C}$, was used until $3 \mathrm{~d}$ after preparation.

The method of yogurt preparation was based on a laboratory-scale manufacturing process commonly conducted at the Food Research and Development Center of Meiji Dairies Corp. The yogurt mix used in this study, containing $0.1 \%$ (wt/wt) fat and $9.5 \%$ (wt/ wt) SNF, was obtained by mixing skim milk powder and water. The materials were supplied by the plants of the Meiji Dairies Corp. For coculture assay in the presence of formic acid or peptides, a predetermined amount of $0.5,1,2$, or $5 \mathrm{~m} M$ sodium formate (Wako Pure Chemical Industries Ltd., Japan) or $0.1 \%$ (wt/wt) casein peptides (CE90GMM, Nippon Shinyaku., Ltd., Japan) was added into the yogurt mix. The yogurt mix was heated to $95^{\circ} \mathrm{C}$ for $2 \mathrm{~min}$ and immediately cooled to $43^{\circ} \mathrm{C}$. The yogurt bulk starter LB81, L. bulgaricus 2038 preculture or Strep. thermophilus 1131 preculture was inoculated in the yogurt mix to a concentration of $2 \%$. After mixing, $15 \mathrm{~g}$ of the mixture was placed into ten 25 -mL glass test tubes. Fermentation was carried out at $43^{\circ} \mathrm{C}$. The yogurt fermentation end point was set at $0.7 \%$ acidity. The yogurt fermentation time was the time required for the original acidity of $0.2 \%$ to change to the end point of $0.7 \%$.

The DO reduction process was as follows: after yogurt starter culture inoculation, sterile nitrogen gas (filtered with a $0.45-\mu \mathrm{m}$ cellulose acetate filter) was aseptically mixed and dispersed into the yogurt mix through a stainless steel pipe (approximately 3 -mm bore) to re- 
duce the DO concentration in the yogurt mix to almost $0 \mathrm{mg} / \mathrm{kg}$. The concentration of DO in the yogurt mix was measured with a DO meter (DO-24P; DKK-TOA Corp., Tokyo, Japan). Yogurt fermentation was carried out at $43^{\circ} \mathrm{C}$ both after the DO reduction treatment and without prior treatment. Yogurt fermentation at $43^{\circ} \mathrm{C}$ without prior treatment was referred to as the control fermentation. Yogurt fermentation after the reduced DO treatment at $43^{\circ} \mathrm{C}$ was referred to as the ROF. The concentration of formic acid in the yogurt mix was measured with a formic acid enzymatic kit (R-Biopharm AG, Darmstadt, Germany).

As shown in Figure 1, the acid production by LB81 at $43^{\circ} \mathrm{C}$ was promoted by reducing the $\mathrm{DO}$ concentration in the yogurt mix to almost $0 \mathrm{ppm}$ (ROF). The time required for the acidity in the yogurt mix to reach $0.7 \%$ was approximately $30 \mathrm{~min}$ shorter than in the control fermentation at $43^{\circ} \mathrm{C}$. However, acid production by L. bulgaricus 2038 or Strep. thermophilus 1131 alone was neither suppressed nor advanced by ROF. Both acid production by L. bulgaricus 2038 alone added 1 $\mathrm{m} M$ sodium formate and Strep. thermophilus 1131 alone added $0.1 \% \mathrm{wt} / \mathrm{wt}$ casein peptides were advanced.

In a previous paper (Horiuchi et al., 2009), we suggested that acid production by LB81 was advanced but that acid production by single culture $L$. bulgari-

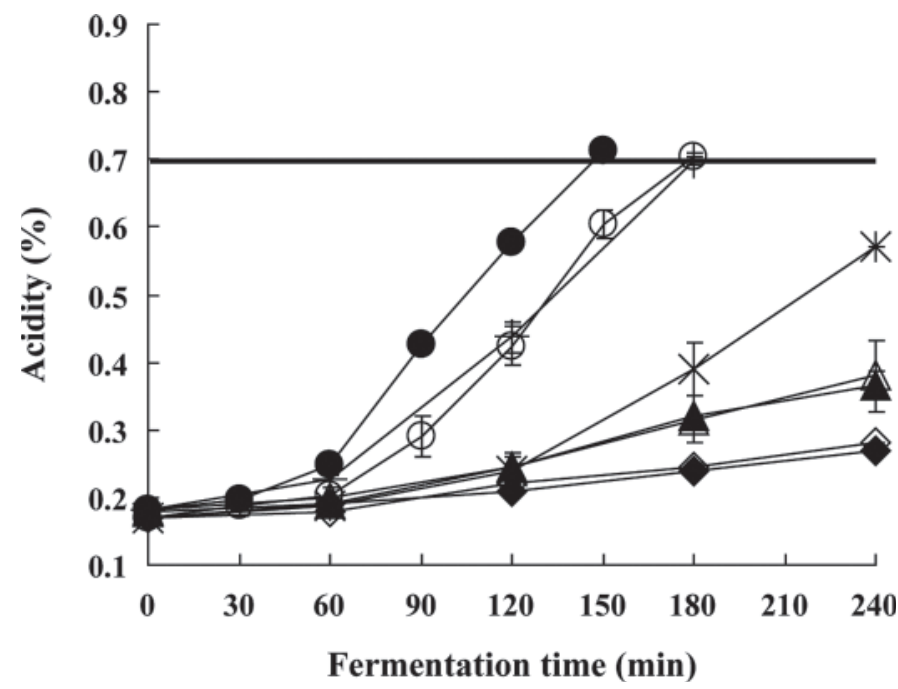

Figure 1. Effect of reduced dissolved oxygen fermentation (ROF) on acid production by the yogurt culture LB81 [composed of Lactococcus delbrueckii ssp. bulgaricus (L. bulgaricus) 2038 and Streptococcus thermophilus 1131] and influence of added sodium formate, added casein peptides, and ROF on acid production by the single yogurt culture $L$. bulgaricus 2038 and Strep. thermophilus 1131. Incubation was at $43^{\circ} \mathrm{C}$. $\mathrm{O}=\mathrm{LB} 81$ without ROF and $\bullet=$ with ROF; $\Delta=$ Strep. thermophilus 1131 without ROF, $\boldsymbol{\Delta}=$ with $\mathrm{ROF}$, and $+=$ added $0.1 \%$ (wt/wt) casein peptides; $\diamond=L$. bulgaricus 2038 without ROF, $\bullet=$ with ROF, and $*$ added $1 \mathrm{~m} M$ sodium formate. Bars represent standard deviations of averages from 3 experiments.

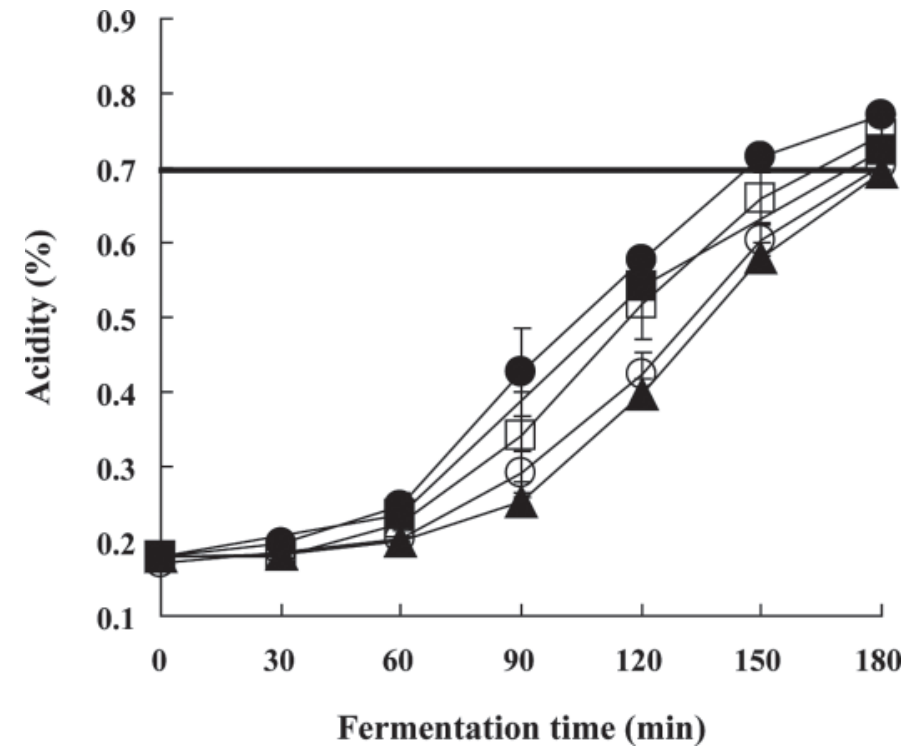

Figure 2. Effect of added sodium formate, added casein peptides, and reduced dissolved oxygen fermentation (ROF) on acid production by the yogurt culture LB81 [composed of Lactococcus delbrueckii ssp. bulgaricus (L. bulgaricus) 2038 and Streptococcus thermophilus 1131]. Incubation was at $43^{\circ} \mathrm{C}$. $\bullet=$ with $\mathrm{ROF} ; \boldsymbol{\bullet}=$ added $1 \mathrm{~m} M$ sodium formate; $\boldsymbol{\Delta}=$ added $0.1 \%$ (wt/wt) casein peptides; $\boldsymbol{\nabla}=$ added $1 \mathrm{~m} M$ sodium formate and $0.1 \%$ (wt/wt) casein peptides; and $\bigcirc=$ control. Bars represent standard deviations of averages from 3 experiments.

cus 2038 or Strep. thermophilus 1131 alone was not advanced or lowered by ROF. From these results, it could be concluded that the DO does not interfere with the growth of single cultures of L. bulgaricus 2038 and Strep. thermophilus 1131 but interferes with the symbiotic relationship between L. bulgaricus 2038 and Strep. thermophilus 1131. Then, in this study, we investigated the influence of DO on the symbiosis between L. bulgaricus and Strep. thermophilus, aiming at growth promoting factors for formic acid.

The effect of formate or casein peptides on acid production velocity by LB81 or single yogurt cultures (Strep. thermophilus 1131 or L. bulgaricus 2038) was examined. As shown in Figure 2, whereas the addition of $1 \mathrm{~m} M$ formate stimulated the acid production by LB81, casein peptides $(0.1 \% \mathrm{wt} / \mathrm{wt})$ had almost no effect on the acidification rate of LB81. As shown in Figure 3A, the acid production by L. bulgaricus 2038 was promoted greatly with $0.5 \mathrm{mM}$ (or more) formate. However, the acid production by Strep. thermophilus 1131 was neither suppressed nor advanced by the addition of formate (Figure 3B).

The formate consumption by single yogurt culture $L$. bulgaricus 2038 was examined. As shown in Figure 4, the concentration of sodium formate in the yogurt mix shrank from a predetermined $0.5,1.0,2.0$, and $5.0 \mathrm{mM}$ to about $0.0,0.5,1.2$, and $3.9 \mathrm{~m} M$, respectively after 
240 min of fermentation. These results showed that the consumption of formate by L. bulgaricus 2038 was approximately $0.5 \mathrm{~m} M$ after $180 \mathrm{~min}$ of fermentation.

The formate accumulation by the single yogurt culture Strep. thermophilus 1131 or LB81 was examined. In the case of symbiotic yogurt culture LB81, the starting point of formate accumulation in fermentation with the reduced DO (ROF) was approximately 30 min earlier than in the control, and the amount of formate ac-

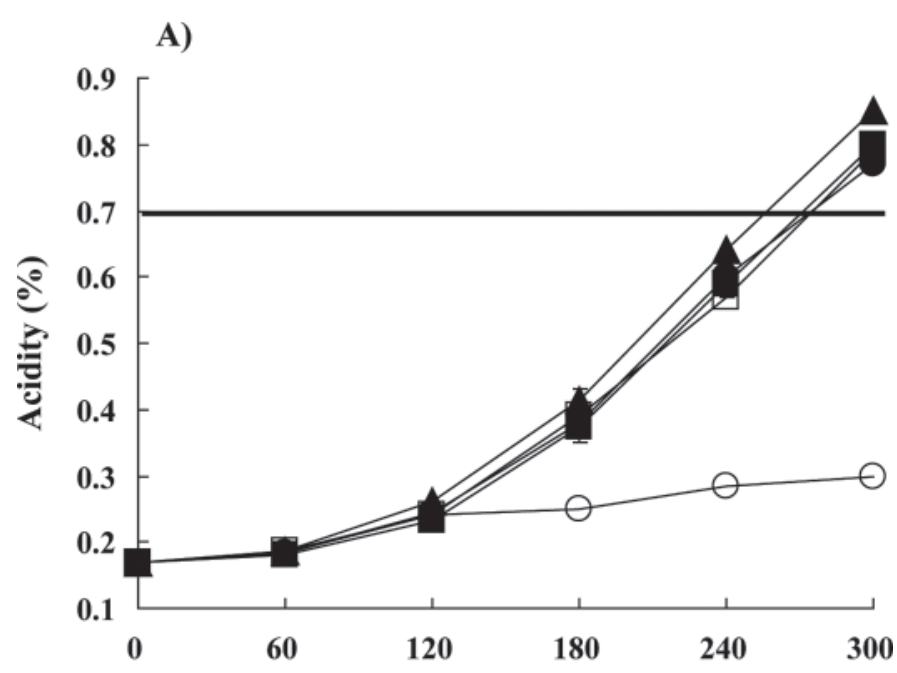

B)

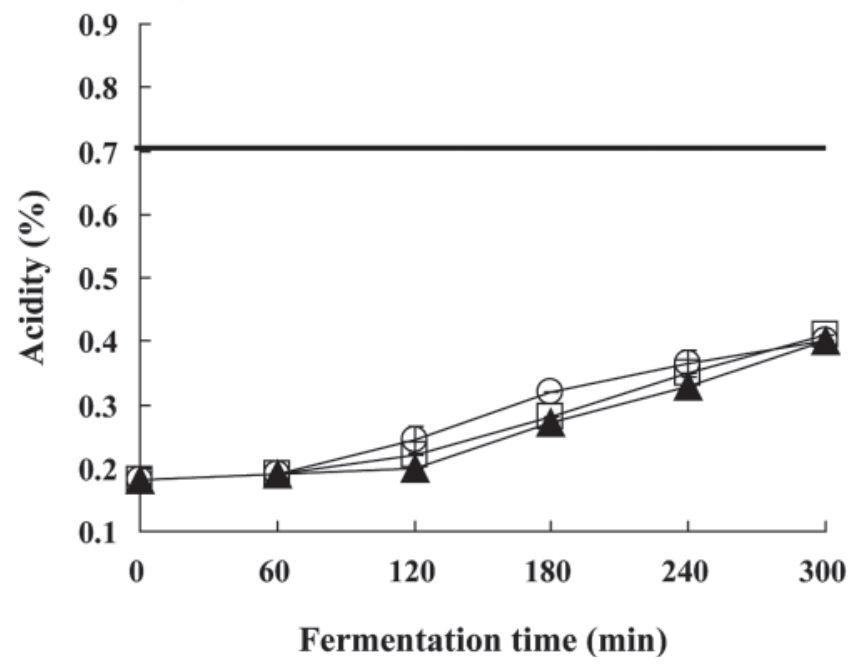

Figure 3. A) Effect of the addition of sodium formate on acid production by the single yogurt culture Lactobacillus delbrueckii ssp. bulgaricus 2038. Incubation was at $43^{\circ} \mathrm{C}$. Sodium formate was added to reach final concentrations of $5(\mathbf{\square}), 2(\mathbf{\Delta}), 1(\bullet), 0.5(\bullet)$, and $0(\bigcirc)$ $\mathrm{m} M$ under normal air conditions. Bars represent standard deviations of averages from 3 experiments. B) Effect of the addition of sodium formate on acid production by the single yogurt culture Streptococcus thermophilus 1131 . Incubation was at $43^{\circ} \mathrm{C}$. Sodium formate was added to reach final concentrations of $2(\boldsymbol{\Lambda}), 1(\diamond)$, and $0(\bigcirc) \mathrm{m} M$ under normal air conditions. Bars represent standard deviations of averages from 3 experiments.

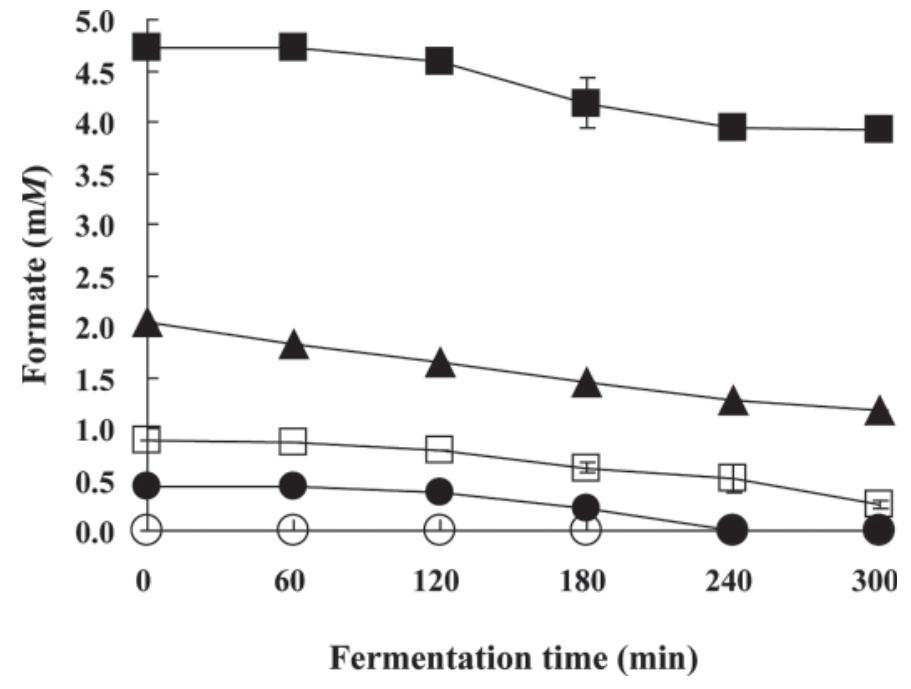

Figure 4. Formate consumption by the single yogurt culture Lactobacillus delbrueckii ssp. bulgaricus 2038 when the sodium formate concentration in the yogurt mix was regulated to approximately $5(\boldsymbol{\square})$, $2(\boldsymbol{\Delta}), 1(\diamond), 0.5(\bullet)$, and $0(\bigcirc) \mathrm{m} M$ beforehand under normal air conditions. Incubation was at $43^{\circ} \mathrm{C}$. Bars represent standard deviations of averages from 3 experiments.

cumulated after 180 min of fermentation by LB81 was approximately $0.8 \mathrm{~m} M$ (Figure 5 ).

It was shown that the accumulation by symbiotic yogurt cultures LB81 composed of L. bulgaricus 2038 and Strep. thermophilus 1131 was approximately 0.8 $\mathrm{m} M$ after $180 \mathrm{~min}$ of fermentation. This $0.8 \mathrm{~m} M$ formic

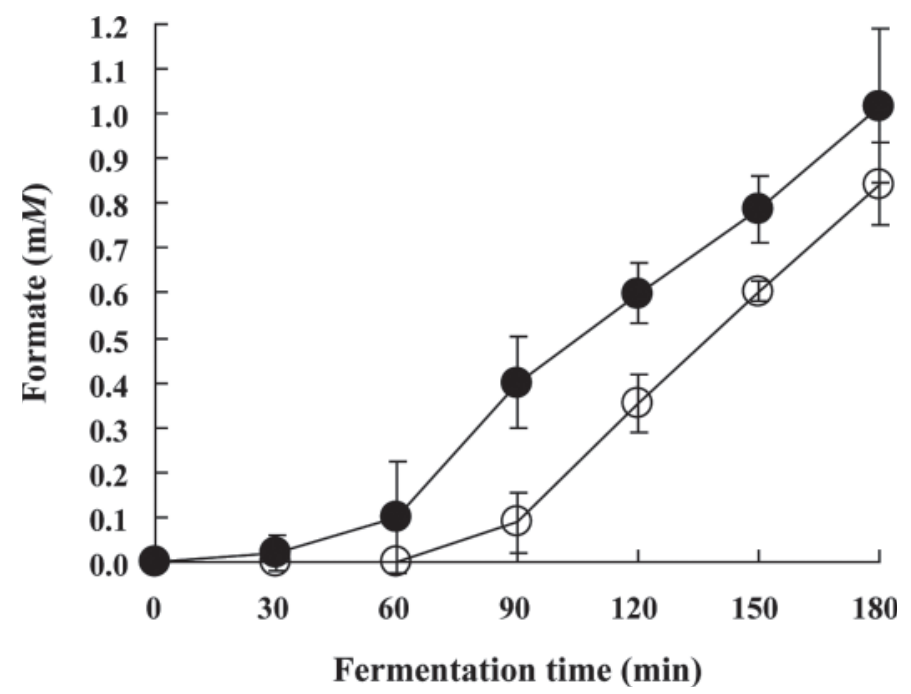

Figure 5. Effect of reduced dissolved oxygen (DO) fermentation (ROF) on formate production by the yogurt culture LB81 [composed of Lactococcus delbrueckii ssp. bulgaricus (L. bulgaricus) 2038 and Streptococcus thermophilus 1131] when the DO concentration in the yogurt mix was regulated to approximately $0 \mathrm{mg} / \mathrm{kg}$ beforehand. Incubation was at $43^{\circ} \mathrm{C}$. - with $\mathrm{ROF}$ and $O=$ without ROF. Bars represent standard deviations of averages from 3 experiments. 
acid was not the production value of formic acid, but the accumulation value of formic acid by LB81 because L. bulgaricus 2038 partly used the formic acid produced by Strep. thermophilus 1131 during fermentation.

It was shown that the formate consumption by single yogurt culture L. bulgaricus 2038 was approximately $0.5 \mathrm{mM}$ and the formate accumulation by symbiotic yogurt cultures LB81 (mixture of Strep. thermophilus 1131 and L. bulgaricus 2038) was approximately 0.8 $\mathrm{m} M$. Although it was not clear how much formate Strep. thermophilus 1131 consumed, from these results, it may be supposed that formate production by LB 81 was approximately $1.3 \mathrm{mM}$.

The acid or formate produced by LB81 with the DO concentration in the yogurt mix fixed at $6,4,2$, and 1 $\mathrm{mg} / \mathrm{kg}$ were examined, adjusting the air or nitrogen gas bubbling into the yogurt mix. Figure 6 shows that acid production by LB81 was delayed in the presence of 1 $\mathrm{mg}$ of $\mathrm{DO} / \mathrm{kg}$ and was suppressed almost completely with more than $2 \mathrm{mg}$ of $\mathrm{DO} / \mathrm{kg}$ in a yogurt mix. Thus, the inhibiting effect of DO on acid production during yogurt fermentation was clearly shown. However, it is not clear how DO, especially at more than $2 \mathrm{mg} / \mathrm{mL}$, inhibits yogurt fermentation, and future studies are necessary to clarify the mechanisms of this inhibition.

As shown in Figure 7A, acid production by LB81 was greatly suppressed with $6 \mathrm{mg}$ of $\mathrm{DO} / \mathrm{kg}$ in the yogurt mix, but it was advanced by adding $1 \mathrm{~m} M$ formate and formate production by LB81 with the DO concen-

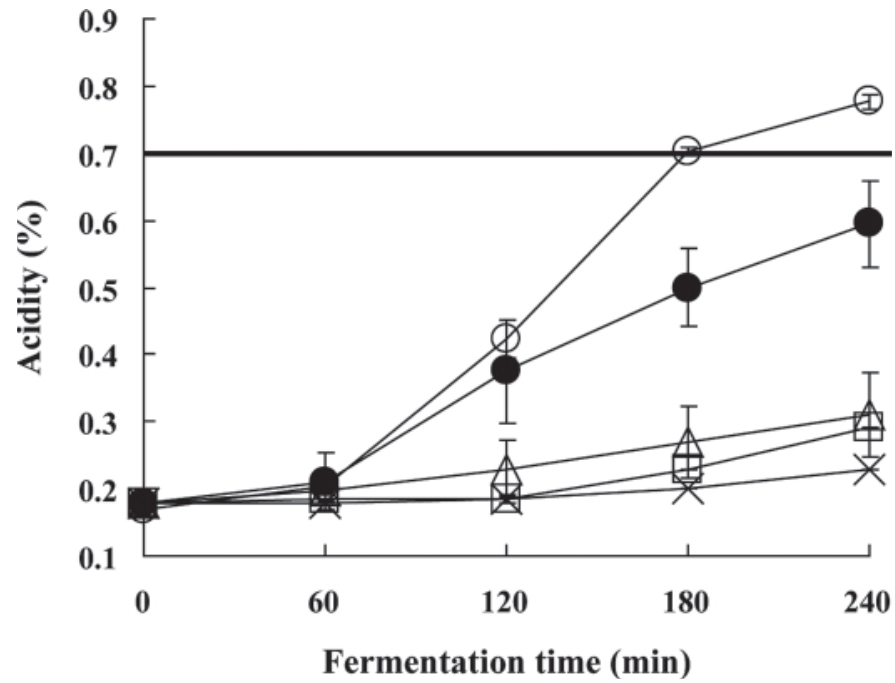

Figure 6. Influence of dissolved oxygen on acid production by the yogurt culture LB81 [composed of Lactococcus delbrueckii ssp. bulgaricus (L. bulgaricus) 2038 and Streptococcus thermophilus 1131]. Incubation was at $43^{\circ} \mathrm{C}$. Dissolved oxygen concentration was fixed at 6 $(\triangle), 4(\times), 3(\square)$, and $1(\bullet) \mathrm{mg} / \mathrm{kg}$, and under normal air conditions (○). Bars represent standard deviations of averages from 3 experiments.

Journal of Dairy Science Vol. 95 No. 6, 2012
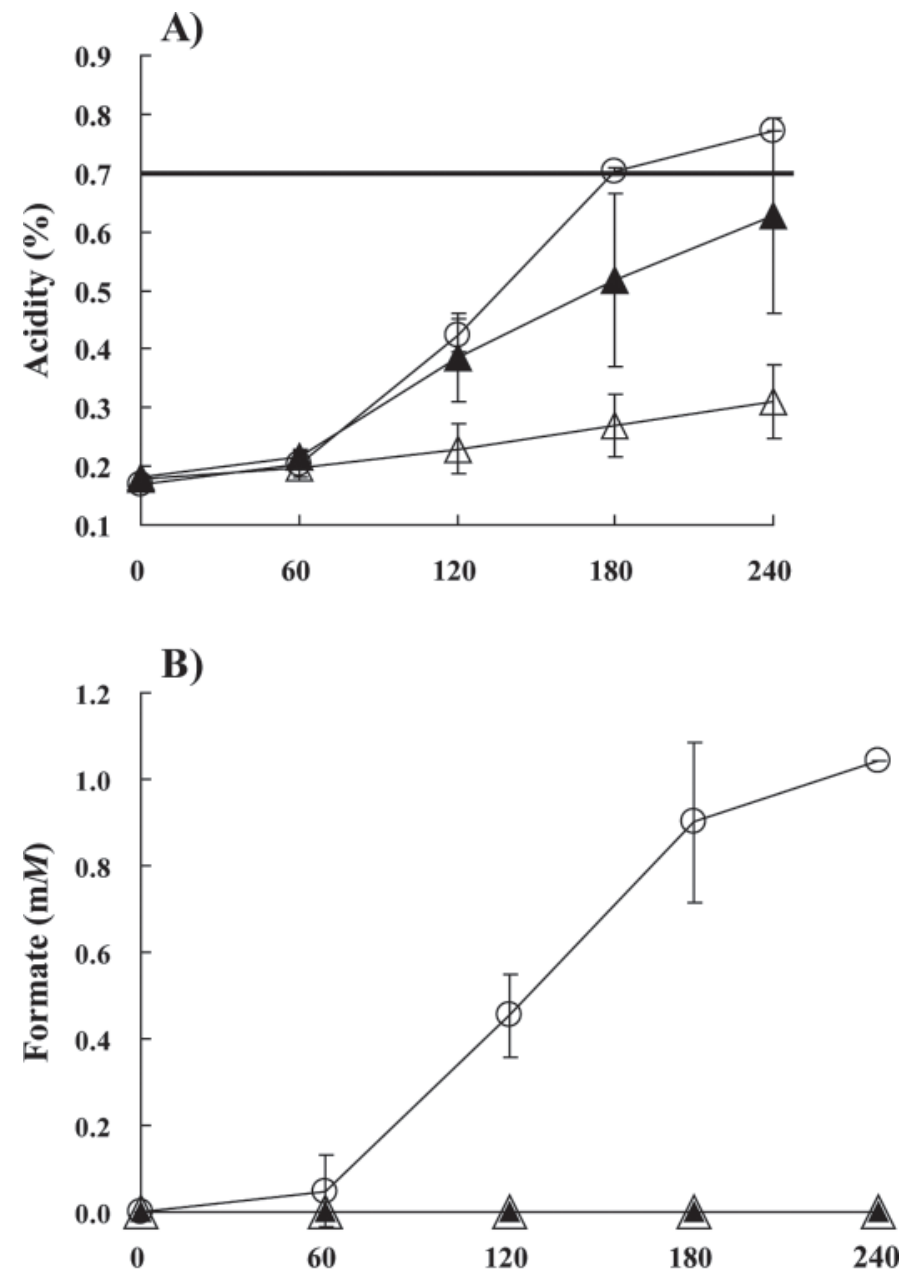

Fermentation time (min)

Figure 7. A) Influence of dissolved oxygen (DO) on acid production by the yogurt culture LB81 [composed of Lactococcus delbrueckii ssp. bulgaricus (L. bulgaricus) 2038 and Streptococcus thermophilus 1131]. Culture LB81 was incubated under a DO concentration fixed at $6 \mathrm{mg} /$ $\mathrm{kg}$. Incubation was at $43^{\circ} \mathrm{C} . \Delta=\mathrm{DO}$ concentration fixed at $6 \mathrm{mg} / \mathrm{kg}$, $\boldsymbol{\Delta}=$ under DO concentration fixed at $6 \mathrm{mg} / \mathrm{kg}$ with $1 \mathrm{~m} M$ formate, and $\bigcirc=$ normal air conditions. Bars represent standard deviations of averages from 3 experiments. B) Influence of dissolved oxygen (DO) on formate production by the yogurt culture LB81. Culture LB81 was incubated under a DO concentration fixed at $6 \mathrm{mg} / \mathrm{kg}$. Incubation was at $43^{\circ} \mathrm{C} . \boldsymbol{\Delta}=\mathrm{DO}$ concentration fixed at $6 \mathrm{mg} / \mathrm{kg}, \Delta=$ DO concentration fixed at $1 \mathrm{mg} / \mathrm{kg}$, and $O=$ normal air conditions. Bars represent standard deviations of averages from 3 experiments.

tration in the yogurt mix fixed at only $1 \mathrm{mg} / \mathrm{kg}$ was completely suppressed (Figure 7B).

It has been shown that Strep. thermophilus produces a growth factor for $L$. bulgaricus under anaerobic or almost anaerobic conditions (Veringa et al., 1968) and this growth factor is equal to or can be replaced by formic acid (Galesloot et al., 1968). In our previous paper, fermentation with LB81 was suppressed by the presence of more than $1 \mathrm{mg}$ of $\mathrm{DO} / \mathrm{kg}$ in the yogurt 
mix. In the present paper, it has been proven that formate production by LB81 with the DO concentration in the yogurt mix fixed at only $1 \mathrm{mg} / \mathrm{kg}$ was completely suppressed. It is generally known that pyruvate formate-lyase (PFL) shows oxygen sensitivity (Knappe et al., 1974) and it was reported that PFL of Escherichia coli or Streptococcus mutans are inactivated by oxygen (Yamada et al., 1985; Sawers and Watson, 1998). We hypothesized that PFL of Strep. thermophilus also shows oxygen sensitivity and it may be concluded that this suppression of acid production was caused by the suppression of formate production.

We investigated the influence of oxygen on the symbiosis between L. bulgaricus and Strep. thermophilus under ROF; normal air conditions; and fixed 6, 4, 2, and $1 \mathrm{mg}$ of $\mathrm{DO} / \mathrm{kg}$ conditions. The results of ROF was almost 30-min earlier formate detection than in normal fermentation (Figure 5) and the addition of formatestimulated acid production by LB81 (Figure 2) showed that the effect of the fermentation time crunch by ROF mainly was the acceleration of formate production of Strep. thermophilus. The result that the fermentation progress by ROF was a little faster than that by addition of formate (Figure 2) suggested that the effect of ROF on fermentation time included a factor(s) other than formate. Acid production by L. bulgaricus 2038 or Strep. thermophilus 1131 in single culture was neither suppressed nor advanced by the DO concentration in the yogurt mix (Figure 1). Although acid production by starter culture LB81 composed of L. bulgaricus 2038 and Strep. thermophilus 1131 was greatly accelerated by reducing DO to almost $0 \mathrm{mg} / \mathrm{kg}$ in the yogurt mix (ROF), both acid production and formate production by LB81 was suppressed by the existence of more than $1 \mathrm{mg}$ of $\mathrm{DO} / \mathrm{kg}$ in the yogurt mix. In conclusion, these results suggest that DO inhibits the symbiotic relationship between L. bulgaricus 2038 and Strep. thermophilus 1131. We attributed the acceleration of acid production of LB81 by reduced DO mainly to the acceleration of formate production and the suppression of acid production of LB81 by DO mainly to the suppression of formate production.

\section{ACKNOWLEDGMENTS}

The authors thank Youichi Niimura and Shinji Kawasaki (Department of Bioscience, Tokyo University of Agriculture, Tokyo, Japan) for their valuable discussions and encouragement, Nobuko Inoue (Research and Development Laboratories, Meiji Co. Ltd., Kanagawa, Japan) for her skilled technical assistance, and Hiroko
Kawashima (Research and Development Laboratories, Meiji Co. Ltd.) for her help in preparing the original manuscript.

\section{REFERENCES}

Condon, S. 1987. Response of lactic acid bacteria to oxygen. FEMS Microbiol. Rev. 46:269-280.

Driessen, F. M., F. Kingma, and J. Stadhouders. 1982. Evidence that Lactobacillus bulgaricus in yogurt is stimulated by carbon dioxide produced by Streptococcus thermophilus. Neth. Milk Dairy J. $36: 135-144$.

FAO/WHO (Food and Agriculture Organization of the United Nations/World Health Organization). 1984. Code of Principles Concerning Milk and Milk Products: International Standards for Milk Products and International Individual Standards for Cheese. Joint FAO/WHO Food Standards Programme. Codex Alimentarius Commission, FAO/WHO, Rome, Italy.

Galesloot, T. H. E., F. Hassing, and H. A. Veringa. 1968. Symbiosis in yoghurt. I. Stimulation of Lactobacillus bulgaricus by a factor produced by Streptococcus thermophilus. Neth. Milk Dairy J. 22:50-63.

Herve-Jimenez, L., I. Guillouard, E. Guedon, S. Boudebbouze, P. Hols, V. Monnet, E. Maguin, and F. Rul. 2009. Postgenomic analysis of Streptococcus thermophilus cocultivated in milk with Lactobacillus delbrueckii subsp. bulgaricus: Involvement of nitrogen, purine, and iron metabolism. Appl. Environ. Microbiol. 75:2062-2073.

Horiuchi, H., N. Inoue, E. Liu, M. Fukui, Y. Sasaki, and T. Sasaki. 2009. A method for manufacturing superior set yogurt under reduced oxygen conditions. J. Dairy Sci. 92:4112-4121.

Kawasaki, S., T. Mimura, T. Satoh, K. Takeda, and Y. Niimura. 2006. Response of the microaerophilic Bifidobacterium species, $B$. boum and B. thermophilum, to oxygen. Appl. Environ. Microbiol. 72:6854-6858.

Knappe, J., H. P. Blaschkowski, P. Gröbner, and T. Schmitt. 1974. Pyruvate formate-lyase of Escherichia coli: The acetyl-enzyme intermediate. Eur. J. Biochem. 50:253-263.

Michaylova, M., S. Minkova, K. Kimura, T. Sasaki, and K. Isawa. 2007. Isolation and characterization of Lactobacillus delbrueckii ssp. bulgaricus and Streptococcus thermophilus from plants in Bulgaria. FEMS Microbiol. Lett. 269:160-169.

Perez, P., G. L. de Antoni, and M. C. Añon. 1991. Formate production by Streptococcus thermophilus cultures. J. Dairy Sci. 74:28502854.

Sakamoto, M., and K. Komagata. 1996. Aerobic growth of and activities of NADH oxidase and NADH peroxidase in lactic acid bacteria. J. Ferment. Bioeng. 82:210-216.

Sawers, G., and G. Watson. 1998. A glycyl radical solution: Oxygendependent interconversion of pyruvate formate-lyase. Mol. Microbiol. 29:945-954.

Shekar, S., and G. S. Bhat. 1983. Influence of dissolved oxygen on acid production in buffalo milk by lactic cultures. J. Food Prot. 46:321-324.

Suzuki, I., S. Kato, T. Kitada, N. Yano, and T. Morichi. 1986. Growth of Lactobacillus bulgaricus in milk. 1. Cell elongation and the role of formic acid in boiled milk. J. Dairy Sci. 69:311-320.

Tamime, A. Y., and R. K. Robinson. 1999. Yoghurt Science and Technology. 2nd ed. Woodhead Publishing Ltd., Cambridge, UK.

Veringa, H. A., T. E. Galesloot, and H. Davelaar. 1968. Symbiosis in yoghurt. II. Isolation and identification of a growth factor for Lactobacillus bulgaricus produced by Streptococcus thermophilus. Neth. Milk Dairy J. 22:114-120.

Yamada, T., S. Takahashi-Abbe, and K. Abbe. 1985. Effects of oxygen on pyruvate formate-lyase in situ and sugar metabolism of Streptococcus mutans and Streptococcus sanguis. Infect. Immun. $47: 129-134$. 